Western University

Scholarship@Western

Physical Therapy Publications

Physical Therapy School

$4-1-2018$

\title{
Descriptive Report of the Impact of Fatigue and Current Management Strategies in Cerebral Palsy.
}

Laura K Brunton

Follow this and additional works at: https://ir.lib.uwo.ca/ptpub

Part of the Physical Therapy Commons

Citation of this paper:

Brunton, L. K. (2018). Descriptive Report of the Impact of Fatigue and Current Management Strategies in Cerebral Palsy. Pediatric Physical Therapy, 30(2), 135-141. 
Fatigue in Cerebral Palsy: Description of Impact and Fatigue Management

Conflict of Interest: The author declares no conflict of interest.

Grant Support: This project was funded by a Canadian Institutes of Health Research Doctoral Scholarship awarded to Laura Brunton for completion of her PhD at Western University in London, Ontario, Canada. 
Abstract

Purpose: Describe the impact of fatigue and self-management practices for adolescents and young adults with cerebral palsy (CP).

Methods: Survey of 124 people with CP with the Fatigue Impact and Severity SelfAssessment (FISSA).

Results: People in GMFCS Level I experienced little impact of fatigue with high proportions of this group disagreeing to statements about fatigue impacting their general activities, mobility and social activities. People in GMFCS Levels II-V reported impact of fatigue on activities. Differences between groups were evident in questions related to fatigue interference with length of time for physical activity and with motivations to participate in social activities. All other items related to management of fatigue were not significantly different between groups.

Conclusions: Fatigue impact is greater for people with more functional limitations. Lack of significant differences between groups on the Management and Activity Modification subscale, indicates more research is needed regarding strategies for fatigue management. 
Fatigue is a common secondary impairment associated with cerebral palsy (CP) $)^{1-2}$ and may be related to changes or declines in physical function, including the cessation of walking. ${ }^{3}$ Adolescents and adults with $\mathrm{CP}$ reportedly experience greater fatigue than the general population..$^{4-7}$ Estimates vary with from 18 to 39\% of adults with CP (aged 16 to 80 years) experiencing fatigue related to CP. Those who are fatigued, have between 12 to $41 \%$ severe fatigued, as measured by a Fatigue Severity Scale (FSS) score $\geq 5.1 .^{5-7}$ The FSS was developed to measure fatigue of people with multiple sclerosis and systemic lupus erythematosus. The scale is 9 questions rated on a 7-point Likert scale. ${ }^{8}$ The FSS has not been validated for use with people with CP. Studies have used the FSS to provide evidence that fatigue is an issue for people with $\mathrm{CP}$ as it is a quick tool and has been used for people who have other neurological conditions. The FSS has limitations, for example, it does not measure self-perceived severity of fatigue. It fails to adequately describe the impact that fatigue may have on an person's life. Finally, the FSS lacks descriptive information about possible targets for intervention or methods that may be useful in managing fatigue.

There are numerous published fatigue scales that have been used with people with neurological conditions, however, until recently there was no fatigue measure validated for use with people with CP. As a result, the Fatigue Impact and Severity Self-Assessment (FISSA) was specifically created and validated for use with people with CP to address the shortcomings of other measurement tools. ${ }^{9}$ The FISSA was created with specific intentions to both identify people with CP-related fatigue and to facilitate discussions between people with $\mathrm{CP}$, families and clinicians about possible management strategies to mitigate the impact of fatigue on daily activities. ${ }^{9}$ The FISSA is a comprehensive self-report questionnaire of 37 items created from a literature review, qualitative interviews with 
people with $\mathrm{CP}$ and focus groups with healthcare providers. ${ }^{9}$ The first 31 questions are scored using a five-point Likert scale from 1 (completely disagree) to 5 (completely agree), ${ }^{9}$ with the exception of items 13-17 which are scored on different scales as they relate to levels of fatigue experienced or amount of time fatigue is experienced. There are 2 subscales; the Impact subscale, with questions on the impact and severity, (items 1-17) and the Management and Activity Modification subscale, with items on possible strategies for mitigating fatigue or consequences of fatigue (items 18-31)..$^{9}$ There are 6 questions that are not scored as part of the measure but ask about diurnal fatigue and open-ended questions about self-management of fatigue for intervention planning. ${ }^{9}$ These questions provide a useful starting point for a conversation about fatigue management (items $32-37$ ). ${ }^{9}$

An impact score is the sum of the first 17 items in the Impact Subscale (including the severity profile) and a separate management score summing the remaining items (items 18-31 in the Management and Activity Modification subscale). ${ }^{9}$ The sum of 31 items is a total fatigue score representing fatigue experienced; considering impact, severity and individual management. The FISSA is a valid and reliable tool for assessing fatigue in people with CP and it defines fatigue as "physical tiredness, muscle soreness, exhaustion of your muscles and body or any related feeling". ${ }^{9}$ Discrimination between groups expected to have more fatigue based on functional ability and pain experiences is evidence of construct validity. ${ }^{9}$ The FISSA has adequate test-retest reliability ICC $(3,1)=0.74$ (95\% CI 0.53-0.87). ${ }^{9}$

The purpose of this study is to describe the impact of fatigue and self-management of adolescents and young adults with CP. The primary objective is to describe and compare responses to the questions on the Impact subscale and Management and Activity Modification subscale of the FISSA between two functionally defined groups; people in 
Gross Motor Function Classification System (GMFCS) ${ }^{10}$ Level I and those in GMFCS Levels II-V.

\section{Method}

This was a descriptive cross-sectional study in which a total of 367 youth and young adults with CP were contacted by regular post or electronic mail as part a larger study. ${ }^{8}$ Participants were recruited from children's rehabilitation centers in Ontario, Canada, previous research studies, pre-existing Facebook groups for people with CP and through an advertisement in the Ontario Federation for Cerebral Palsy website and newsletter. Eligibility criteria included aged 14 to 31 years, with a diagnosis of CP (self-reported), who were English speaking and had the ability to complete self-report questionnaires with a degree of independence. Surveys completed entirely by parental proxy were excluded from the study. The mailing contained the FISSA, a self-report version of the GMFCS-Extended and Revised Version ${ }^{11}$ to describe the functional abilities of respondents (self-report version available from https://www.canchild.ca) and a demographic questionnaire used to obtain self-reported distribution of impaired body regions, age, sex and information regarding the amount of assistance required to complete the survey. The study used a modified Dillman approach, ${ }^{12}$ in which participants were contacted either 2 or 3 times depending on their participation. All potential participants initially received a full survey package (or an email with the letter of information and survey link) containing a $\$ 10$ gift card or code as an incentive to participate. In an effort to increase the number of respondents, a reminder letter was mailed to each potential participant approximately 2 weeks following the initial package mailing. All potential participants who had not returned the survey 2 weeks after the reminder letters were sent a second full questionnaire 
package 4 weeks following the initial mailing. A staff members at the children's rehabilitation centers facilitated the mailing of surveys and reminder letters as per their recruitment policies. The investigator facilitated mailing for participants recruited from other sources. All returned questionnaires were anonymous through the use of a study ID number. This study received approval from the ethics review boards at Western University, McMaster University and specific clinical sites as appropriate. Returning a completed questionnaire was considered implied consent to participate in this study.

As a result of the small number of participants in each GMFCS Level, people were grouped together to increase the subgroup sample size. Two groups were created, the first group consisted of people at GMFCS Level I (walk without limitations). The second group consisted of people at GMFCS Level II (walk with limitations), Level III (walk using a handheld mobility device) Level IV (use powered mobility due to limitations in selfmobilization) and Level $V$ (without the ability to self-mobilize). ${ }^{11}$ These 2 groups were based on previous sub-studies of the same dataset that indicated the total fatigue (FISSA) score was not different among those with ambulatory limitations (GMFCS II-V) but those groups were significantly different from those in Level I. ${ }^{9,13}$. In a previous study with this dataset, the FISSA scores (Impact subscale and Management subscale scores) differed by the pre-identified GMFCS grouping. ${ }^{13}$ In the current study, descriptive analysis of the survey responses was completed using a Pearson's Chi-Square for each question based on the 2 groups, with a Bonferroni correction to account for the multiple comparisons (significance level for Impact subscale set to $\mathrm{p}<0.0042$, and $\mathrm{p}<0.0036$ for the Management and Activity Modification subscale).

\section{Results}


We received returns from 163 people of the 367people contacted (response rate of 44\%). Thirty-three returned questionnaires were excluded from analysis because they were either returned blank, or were from people who did not meet eligibility criteria or were completed entirely by parental proxy. An additional six participants failed to report their GMFCS level and were unable to be grouped for analysis. The final convenience sample consisted of 124 adolescents and young adults with CP (response rate of 34\%) who returned a questionnaire completed at least semi-independently (independent completion or having had some assistance completing the questionnaire). Participant demographics are in Table 1.

Table 2 provides the frequency of responses by group for each item on the Impact subscale and Table 3 provides this information for each item on the Management and Activity Modification subscale of the FISSA.

Scores on Impact subscale items were significantly different $(\mathrm{p}<0.0042)$ between the groups (items $1-11 ; \mathrm{p}<0.001$ for items $1-5$ and $8-10, p=0.001$ for items 6,7 and 11 ) except for item 12 "I have had to reduce my responsibilities at home because of fatigue" $(p=0.015)$.

Significant differences on the Management and Activity Modification subscale between groups were only evident in the questions related fatigue interference with the length of time someone could be physically active (item $20 ; p=0.001$ ) and with motivations to participate in social activities (item 23; $\mathrm{p}=0.003$ ). Participants in GMFCS Level I were almost equally split on their thoughts about fatigue interference with the length of time they could be active physically; $41 \%$ disagreed with this statement and $50 \%$ agreed that fatigue impacted the length of physical activity sessions. Of the participants in Levels II-V, 
$85 \%$ agreed that fatigue limited the length of physical activity sessions. Sixty-eight percent at GMFCS Level I disagreed that fatigue impacted their motivation for social interaction, and $49 \%$ of at GMFCS Levels II-V felt fatigue did have an impact on motivation to participate in social activities. The remaining items on the fatigue Management and Activity Modification subscale were not significantly different between the two groups. Despite the difference in impact of fatigue between the 2 groups, participants in both groups equally disagreed with statements that they use specific management strategies.

\section{Discussion}

Participants in GMFCS Level I reported little impact of fatigue on their general activities, independence, self-care and mobility activities. However, people in GMFCS II through $\mathrm{V}$ did report significant impact of fatigue on their general activities, independence and mobility. Only 32\% of those in GMFCS Levels II-V agreed that fatigue impacted their ability to perform self-care activities, likely representing some differences between GMFCS levels contained within this larger grouping. Similarly, about half the participants in Levels II-V reported that they used adaptive equipment to manage fatigue.

Based on the observed differences on items contained in the Impact subscale it is clear that the overall impact of fatigue is greater for people with more functional limitations. The lack of differences between the two groups on the items related to strategies used to mitigate fatigue is not surprising given the lack of interventions aimed at fatigue management for all people with $\mathrm{CP}$ in the current healthcare climate. Many studies have reported the presence of fatigue in people with $\mathrm{CP}$ but there are currently no evidence-based interventions for fatigue management for people with CP. Fatigue management strategies such as pacing, planning, resting and activity modification were 
discussed by participants in a qualitative study regarding the bodily experience of $\mathrm{CP}$ and were therefore included as explicit questions in the Management and Activity Modification subscale of the FISSA to facilitate a better understanding of the strategies used by youth with CP..$^{14}$ In the current study, resting was the most commonly employed strategy with $56 \%$ of the sample reporting that they stop and rest to mitigate fatigue levels. The next most popular strategy used was pacing; however, less than half (44\% of total sample) of the participants reported using this strategy. Finally, planning was the least common fatigue management strategy used by participants in this study. Close to $70 \%$ reported they did not think about fatigue when planning their day. The lack of significant differences for many of the items on the Management and Activity Modification subscale of the FISSA combined with the known difference in impact of fatigue begs the question, are we, as a healthcare community, doing enough to help manage fatigue for people with CP across all levels of functional ability? Strategies such as pacing, planning, resting and activity modification should be integrated into clinical conversations about fatigue management to address the overwhelming impact and individualized experience of fatigue reported by youth with CP. Of note, only $30 \%$ of the full sample reported limiting their physical activity to manage their fatigue. This result is promising as it is thought that increasing physical activity levels may be useful for managing fatigue, $15,16,17$ though the specific relationship between physical activity and fatigue remains unknown. Observations from the current study in combination with the published evidence related to fatigue in people with $\mathrm{CP}$ continue to demonstrate the complexity of the relationship between fatigue and physical activity and highlight the need to better understand this relationship. 
Further exploration of many of the remaining Management and Activity Modification subscale items revealed a great deal of variability in responses that reinforce the highly individualized presentation of $\mathrm{CP}$ and may provide opportunities for increased clinical guidance for fatigue management. For example, many people felt that additional stress in their lives increased their fatigue levels (item 26), or the reverse that experiencing fatigue was stressful (item 27), while others didn't agree with these statements. Clinicians could explore stress reducing strategies for people who agree with these items on the FISSA as a potential fatigue management intervention. Similarly, motivation to participate in physical (item 22), and social activities (item 23) was quite variable, as was the effect of fatigue on participation in leisure and recreation activities (item 19). This variability in responses within and across functional ability levels may represent the value that different people place on being social, physically active, or the enjoyment they receive from participating in leisure activities and could be a potential target for clinical intervention.

The FISSA is a tool that can be used to initiate or guide clinical conversations, in alignment with current rehabilitation practices using shared management approaches to care. ${ }^{9}$ The use of the FISSA may allow for a better understanding of the variable nature of fatigue in people with $\mathrm{CP}$, and as it pertains to a specific client. For example, in the current study, approximately half the sample (49\%) felt that long periods of inactivity increased their fatigue. In a clinical setting, the Canadian 24-hour movement guidelines ${ }^{18}$ or the recently published physical activity guidelines for people with $\mathrm{CP}^{19}$ could be used as educational tools to facilitate conversations about fatigue management. For example, clinicians may suggest small, manageable bouts of physical activity to decrease sedentary behaviours and mitigate fatigue for people who agree with this item on the FISSA. 
As another example, the participants in the current study were overwhelmingly in agreement that fatigue can interfere with balance and coordination (item 21). Clinicians know that when balance and coordination are affected there may be an increased risk of experiencing a fall and/or sustaining an injury that may affect functional status. Therefore, adequate fatigue management, ideally self-awareness and self-management of fatigue guided by a rehabilitation clinician, may reduce the negative health consequences of falls or unintentional injuries and contribute to greater overall health and wellbeing across the lifespan.

Early identification of fatigue and timely intervention should be a health promotion goal that rehabilitation professionals discuss with families and people with CP throughout the adolescent years. Given the impact that fatigue can have on functional status in adulthood, ${ }^{3}$ we need to capitalize on the supportive care models provided in the pediatric healthcare setting. Early recognition and intervention are more feasible during the childhood and adolescent years, because the supports that exist for people transitioning into adulthood are variable and often non-existent.

\section{Limitations}

This study used a convenience sampling approach that may have resulted in a selection bias. It is possible that people experiencing fatigue were more likely to respond to a survey about fatigue and this could result in an over-estimation of the fatigue experienced by the population with CP. In addition, the treatment centers used to identify potential participants required an internal staff member to facilitate the survey mailing, as a result the author did not have access to descriptors for people who did not respond to the survey mailing. The author was therefore unable to determine if the characteristics of 
responders and non-responders were different, potentially affecting the generalizability of the findings from this study. The small sample size in the current study prevented the possibility of looking at the GMFCS levels independently and required collapsing people in GMFCS levels II-V into a single group which may limit the interpretations of the results of this study, however, this is the first study to describe these important characteristics of fatigue for this population as a whole.

\section{Future Directions}

Given the paucity of knowledge about fatigue in people with $\mathrm{CP}$, ongoing research is needed. Future studies should focus on identifying the relative age of onset and peak age of fatigue impact and severity. In addition, researchers should track these outcomes over time and with growth and development. Studies with larger sample sizes and power to detect differences at single GMFCS levels would be beneficial in exploring the fatigue patterns related to functional abilities, for instance people who are independent in ambulation versus those who use gait aids. In addition, a greater understanding of what people are currently doing to self-manage fatigue in the context of their daily lives is integral to supporting these people to participate in their life roles. Finally, interventions to address fatigue need to be developed, implemented and evaluated, as there are currently no evidence-informed fatigue management practices for rehabilitation clinicians to offer their clients with $\mathrm{CP}$.

Qualitative data from the six additional, open-ended, questions may provide more information about the management strategies used by (or considered but not implemented) people with CP. These data are available and will be analyzed and presented separately to further the literature related to fatigue management for people with CP. 


\section{Conclusion}

This paper is the first to report descriptive information on fatigue experienced by people with CP using a validated measure. There was a significant difference observed between the two groups for the impact of fatigue on daily life, such that fatigue had a higher impact for people with more functional limitations. The absence of observed differences between the two groups on the Management and Activity Modification subscale, may be due to the lack of available fatigue management strategies to people with $\mathrm{CP}$, regardless of functional ability level. It is clear that there is a need to continue to explore and understand the causes, impact and severity of fatigue experienced by these people to provide meaningful and relevant fatigue interventions for management of this secondary condition. Fatigue is a commonly reported cause of functional deterioration; better prevention and management could increase the quality of life for people with CP of all ages and functional ability levels.

\section{Acknowledgements}

I would like to thank Dr. Doreen Bartlett for her support of and guidance on this project and her careful review of this manuscript. 


\section{References}

1. Hilberink SR, Roebroeck ME, Nieuwstraten W, et al. Health issues in young adults with cerebral palsy: towards a life-span perspective. J Rehabil Med. 2007;39:605-611.

2. Maher $\mathrm{C}$, Crettenden A, Evans $\mathrm{K}$, et al. Fatigue is a major issue for children and adolescents with physical disabilities. Dev Med Child Neurol. 2015;57:742-747.

3. Jahnsen R, Villien L, Egeland T, et al. Locomotion skills in adults with cerebral palsy. Clin Rehabil. 2004;18:309-316.

4. Jahnsen R, Villien L, Stanghelle JK, et al. Fatigue in adults with cerebral palsy in Norway compared with the general population. Dev Med Child Neurol. 2003;45:296-303.

5. Slaman J, Bussmann J, van der Slot WM, et al. Physical strain of walking relates to activity level in adults with cerebral palsy. Arch Phys Med Rehabil. 2013;94:896-901.

6. van der Slot WM, Nieuwenhuijsen C, van den Berg-Emons RJ, et al. Chronic pain, fatigue, and depressive symptoms in adults with spastic bilateral cerebral palsy. Dev Med Child Neurol. 2012;54:836-842.

7. Russchen HA, Slaman J, Stam HJ, et al. Focus on fatigue amongst young adults with spastic cerebral palsy. J Neuroeng Rehabil. 2014;11:161-168.

8. Krupp LB, LaRocca NG, Muir-Nash J, et al. The Fatigue Severity Scale: Application to patients with multiple sclerosis and systemic lupus erythematosus. Arch Neurol. 1989;46:1121-3.

9. Brunton L, Bartlett D. Construction and validation of the Fatigue Impact and Severity Self-Assessment for youth and young adults with cerebral palsy. Dev Neurorehabil. Early Online, DOI:10.1080/17518423.2016.1189974.

10. Palisano R, Rosenbaum P, Walter S, et al. Development and reliability of a system to classify gross motor function in children with cerebral palsy. Dev Med Child Neurol. 1997;39:214-223.

11. Palisano RJ, Rosenbaum P, Bartlett D, et al. Content validity of the expanded and revised Gross Motor Function Classification System. Dev Med Child Neurol. 2008;50:744-750.

12. Dillman DA. Mail and internet surveys: The tailored design method. New York: Wiley; 2000.

13. Brunton LK, Bartlett DJ. Profiles of fatigue severity and variability among adolescents and young adults with cerebral palsy. Fatigue. 2016:1-10.

DOI:10.1080/21641846.2017.1264950. 
14. Brunton LK, Bartlett DJ. The bodily experience of cerebral palsy: A journey to selfawareness. Disabil Rehabil. 2013;35(23):1981-90.

15. Tosi LL, Maher N, Moore DW, Goldstein M, Aisen ML. Adults with cerebral palsy: a workshop to define the challenges of treating and preventing secondary musculoskeletal and neuromuscular complications in this rapidly growing population. Dev Med Child Neurol. 2009;51(S4):2-11.

16. McPhee PG, Brunton L, Timmons B, Bentley T, Gorter JW. Examining fatigue in ambulatory and non-ambulatory adults with cerebral palsy: prevalence, impact and relationships with physical activity and body composition. Dev Med Child Neurol. 2017;59(4):367-73. DOI: 10.1111/dmcn.13306

17. Jahnsen R, Villien L, Aamodt G, Stanghelle JK, Holm I. Physiotherapy and physical activity - Experiences of adults with cerebral palsy, with implications for children. $A d v$ Physiother. 2003;5:21-32.

18. Tremblay MS, Carson V, Chaput JP, Connor Gorber S, Dinh T, Duggan M, Janssen I. Canadian 24-Hour Movement Guidelines for Children and Youth: An Integration of Physical Activity, Sedentary Behaviour, and Sleep. Appl Physiol Nutr Metab. 2016;41(6):S311-27.

19. Verschuren O, Peterson MD, Balemans AC, et al. Exercise and physical activity recommendations for people with cerebral palsy. Dev Med Child Neurol. 2016;58:798-808. 
Table 1 - Participant Characteristics and Demographic Information of the Sample

\begin{tabular}{lll}
\hline Characteristic & & $\begin{array}{c}\text { Total (n=124) } \\
\mathbf{n}(\%)\end{array}$ \\
\hline Sex $^{*}$ & Male & $58(47 \%)$ \\
& Female & $65(53 \%)$ \\
Age & Mean, years (SD) & $18.7(4.5)$ \\
& Median & 17 \\
& Range & $14-31$ \\
GMFCS Level & & \\
& I & $34(27 \%)$ \\
& II & $39(32 \%)$ \\
& III & $21(17 \%)$ \\
Distribution of Involvement & $18(14 \%)$ \\
& IV & $12(10 \%)$ \\
& V & \\
& Monoplegia & $6(5 \%)$ \\
& Hemiplegia & $31(25.5 \%)$ \\
& Diplegia & $44(36 \%)$ \\
& Triplegia & $10(8 \%)$ \\
& Quadriplegia & $31(25.5 \%)$ \\
\hline
\end{tabular}

GMFCS = Gross Motor Function Classification System; Note: *one participant did not report their sex; ${ }^{\square}$ two participants did not report distribution of involvement. 
Table 2 - Frequency Responses Between Functional Groups for The Impact Subscale

\begin{tabular}{|c|c|c|c|c|c|c|c|c|c|c|c|}
\hline \multirow{2}{*}{$\begin{array}{c}\text { Response Option } \\
\text { Item (Impact Subscale) }\end{array}$} & \multicolumn{2}{|c|}{$\begin{array}{l}\text { Completely } \\
\text { Disagree }\end{array}$} & \multicolumn{2}{|c|}{$\begin{array}{c}\text { Somewhat } \\
\text { Disagree }\end{array}$} & \multicolumn{2}{|c|}{$\begin{array}{c}\text { Neither Agree } \\
\text { nor Disagree }\end{array}$} & \multicolumn{2}{|c|}{$\begin{array}{c}\text { Somewhat } \\
\text { Agree }\end{array}$} & \multicolumn{2}{|c|}{$\begin{array}{c}\text { Completely } \\
\text { Agree }\end{array}$} & \multirow[b]{2}{*}{$\begin{array}{c}\mathbf{p} \\
\text { value }\end{array}$} \\
\hline & $\begin{array}{c}\text { GMFCS } \\
\text { I }\end{array}$ & $\begin{array}{l}\text { GMFCS } \\
\text { II-V }\end{array}$ & $\begin{array}{c}\text { GMFCS } \\
\text { I } \\
\end{array}$ & $\begin{array}{l}\text { GMFCS } \\
\text { II-V }\end{array}$ & $\begin{array}{c}\text { GMFCS } \\
\text { I } \\
\end{array}$ & $\begin{array}{l}\text { GMFCS } \\
\text { II-V }\end{array}$ & $\begin{array}{c}\text { GMFCS } \\
\text { I } \\
\end{array}$ & $\begin{array}{c}\text { GMFCS } \\
\text { II-V }\end{array}$ & $\begin{array}{c}\text { GMFCS } \\
\text { I } \\
\end{array}$ & $\begin{array}{c}\text { GMFCS } \\
\text { II-V }\end{array}$ & \\
\hline $\begin{array}{l}\text { 1. Fatigue interferes with } \\
\text { my general everyday } \\
\text { activities }\end{array}$ & $32.4 \%$ & $10.0 \%$ & $47.1 \%$ & $15.6 \%$ & $11.8 \%$ & $10.0 \%$ & $5.9 \%$ & $45.6 \%$ & $2.9 \%$ & $18.9 \%$ & $<0.001$ \\
\hline $\begin{array}{l}\text { 2. Fatigue interferes with } \\
\text { my ability to move around } \\
\text { indoors }\end{array}$ & $64.7 \%$ & $20 \%$ & $29.4 \%$ & $15.6 \%$ & $2.9 \%$ & $12.2 \%$ & $2.9 \%$ & $38.9 \%$ & $0 \%$ & $13.3 \%$ & $<0.001$ \\
\hline $\begin{array}{l}\text { 3. Fatigue interferes with } \\
\text { my ability to do things on } \\
\text { my own }\end{array}$ & $67.6 \%$ & $16.7 \%$ & $11.8 \%$ & $13.3 \%$ & $14.7 \%$ & $18.9 \%$ & $2.9 \%$ & $31.1 \%$ & $2.9 \%$ & $20.0 \%$ & $<0.001$ \\
\hline $\begin{array}{l}\text { 4. Fatigue interferes with } \\
\text { my ability to move around } \\
\text { in my community }\end{array}$ & $47.1 \%$ & $18.9 \%$ & $26.5 \%$ & $12.2 \%$ & $20.6 \%$ & $20.0 \%$ & $2.9 \%$ & $25.6 \%$ & $2.9 \%$ & $23.3 \%$ & $<0.001$ \\
\hline $\begin{array}{l}\text { 5. Fatigue interferes with } \\
\text { my ability to get outside of } \\
\text { my house }\end{array}$ & $73.5 \%$ & $26.7 \%$ & $14.7 \%$ & $13.3 \%$ & $5.9 \%$ & $18.9 \%$ & $2.9 \%$ & $25.6 \%$ & $2.9 \%$ & $15.6 \%$ & $<0.001$ \\
\hline $\begin{array}{l}\text { 6. Fatigue interferes with } \\
\text { my ability to finish things }\end{array}$ & $38.2 \%$ & $12.2 \%$ & $20.6 \%$ & $7.8 \%$ & $14.7 \%$ & $14.4 \%$ & $23.5 \%$ & $45.6 \%$ & $2.9 \%$ & $20.0 \%$ & 0.001 \\
\hline $\begin{array}{l}\text { 7. Fatigue interferes with } \\
\text { my participation in social } \\
\text { activities }\end{array}$ & $47.1 \%$ & $17.8 \%$ & $26.5 \%$ & $10.0 \%$ & $11.8 \%$ & $17.8 \%$ & $14.7 \%$ & $36.7 \%$ & $0.0 \%$ & $17.8 \%$ & 0.001 \\
\hline
\end{tabular}


Table 3 - Frequency Responses Between Functional Groups for The Management and Activity Modification Subscale

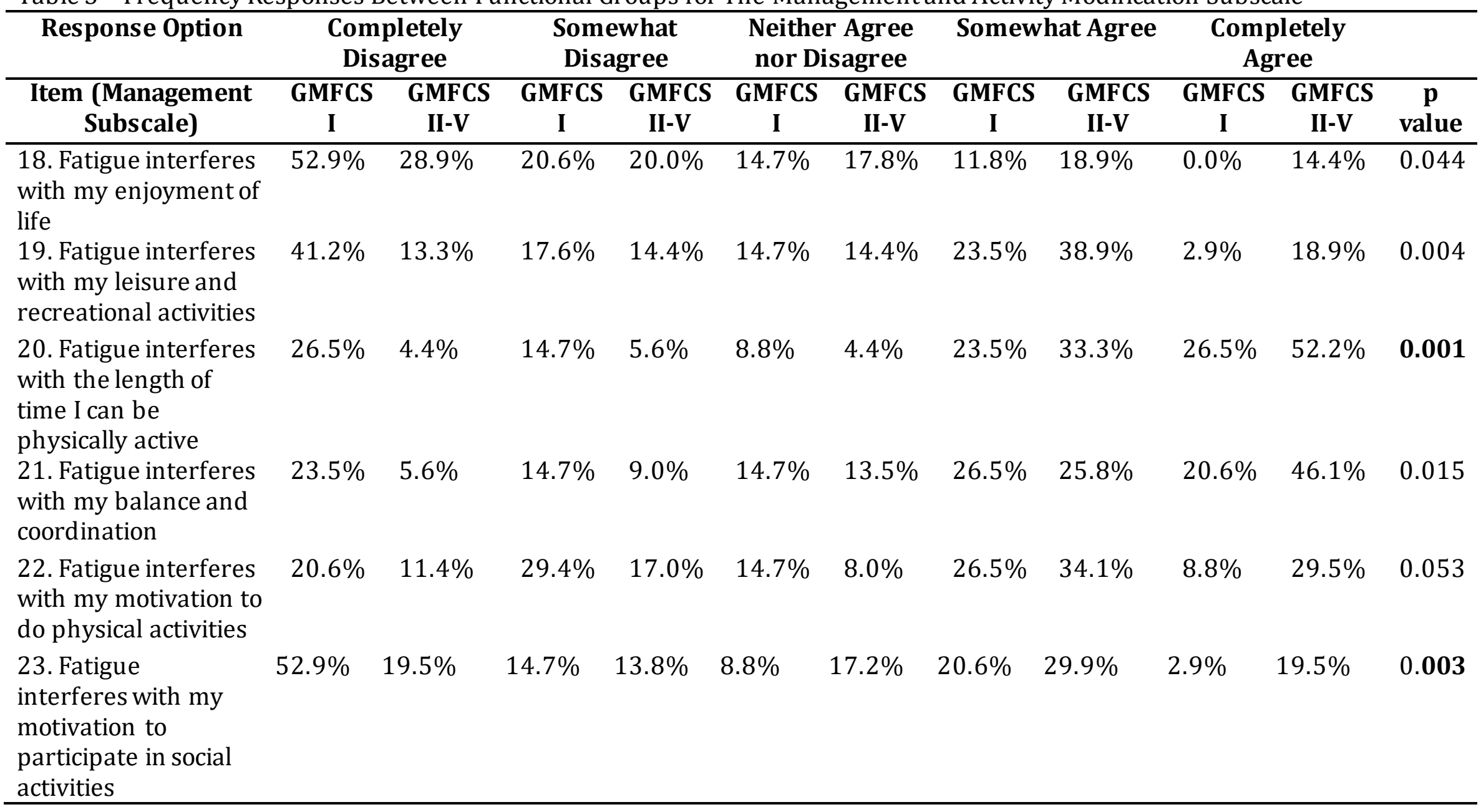

